ANL-HEP-PR-07-98

DESY 07-199

LPT-ORSAY 0\%-109

$M A N / H E P / 2007 / 26$

PITHA $07 / 15$

\title{
Determining Heavy Mass Parameters in Supersymmetric SO(10) Models
}

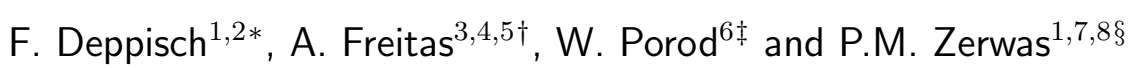 \\ 1 Deutsches Elektronen-Synchrotron DESY, D-22603 Hamburg, Germany \\ 2 School of Physics and Astronomy, University of Manchester, Manchester M13 9PL, UK \\ 3 Inst. Theor. Physik, Universität Zürich, CH-8057 Zürich, Switzerland \\ 4 Enrico Fermi Institute, University of Chicago, Chicago, IL 60637, USA \\ ${ }^{5}$ HEP Division, Argonne National Laboratory, Argonne, IL 60439, USA \\ ${ }^{6}$ Inst. Theor. Physik und Astrophysik, Universität Würzburg, D-97074 Würzburg, Germany \\ 7 Inst. Theor. Physik E, RWTH Aachen, D-52056 Aachen, Germany \\ 8 Laboratoire de Physique Théorique, U. Paris-Sud, F-91405 Orsay, France
}

\begin{abstract}
Extrapolations of soft scalar mass parameters in supersymmetric theories can be used to explore elements of the physics scenario near the grand unification scale. We investigate the potential of this method in the lepton sector of $\mathrm{SO}(10)$ which incorporates right-handed neutrino superfields. The high precision expected from coherent LHC and $e^{+} e^{-}$collider analyses allows the reconstruction of the fundamental scalar mass parameters at the unification scale and the D-terms related to the breaking of grand unification symmetries. In addition, the mass of the third-generation righthanded neutrino can be estimated in seesaw scenarios.
\end{abstract}

\footnotetext{
${ }^{*}$ E-mail: frank.deppisch@manchester.ac.uk

${ }^{\dagger}$ E-mail: afreitas@hep.anl.gov

${ }_{\ddagger}^{\ddagger}$ E-mail: porod@physik.uni-wuerzburg.de

$\S$ E-mail: zerwas@desy.de
} 


\section{Introduction}

The observation of neutrino oscillations has provided experimental proof for non-zero neutrino masses [1]. When right-handed neutrinos, not carrying any Standard Model gauge charges, are included in the set of leptons and quarks, the symmetry group $\mathrm{SO}(10)$ is naturally suggested as the grand unification group [2]. For theories formulated in a supersymmetric framework to build a stable bridge between the electroweak scale and the Planck scale, a scalar R-neutrino superfield is added to the spectrum of the minimal supersymmetric standard model.

A natural explanation of the very light neutrino masses in relation to the electroweak scale is offered by the seesaw mechanism [3]. For right-handed Majorana neutrino masses $M_{\nu_{R i}}$ in a range close to the grand unification (GUT) scale, small neutrino masses can be generated quite naturally by this mechanism: $m_{\nu_{i}} \sim m_{q_{i}}^{2} / M_{\nu_{R i}}$, with $m_{q_{i}}$ denoting up-type quark masses. In parallel, the R-sneutrino masses are very heavy too.

In the present analysis we will first focus on one-step symmetry breaking from $\mathrm{SO}(10)$ down to the Standard Model SM,

(I) $\mathrm{SO}(10) \underset{\Lambda_{\mathcal{U}}}{\rightarrow} \mathrm{SM}$

with $\Lambda_{\mathcal{U}} \approx 2 \cdot 10^{16} \mathrm{GeV}$ denoting the usual GUT scale with apparent unification of the SM gauge couplings in supersymmetric theories. The report expands on earlier work in Ref. [4] by including a systematic analysis of neutrino mixing. In a subsequent section we will add the analysis of a specific two-step breaking chain, cf. Ref. [5],

(II) $\mathrm{SO}(10) \underset{\Lambda_{\mathcal{O}}}{\rightarrow} \mathrm{SU}(5) \underset{\Lambda_{\mathcal{U}}}{\rightarrow} \mathrm{SM}$,

$\Lambda_{\mathcal{O}}>\Lambda_{\mathcal{U}}$ denoting the $\mathrm{SO}(10)$ breaking scale, while $\Lambda_{\mathcal{U}}$ is fixed again by the unification scale of the gauge couplings. Though this extension will be formulated in a simple scheme, it confronts us with consequences of presently unsolved conceptual problems of $\mathrm{SO}(10)$ scenarios in phenomenological analyses more strongly than the one-step scheme. Nevertheless, this hypothetical chain may serve as an interesting example for coping with the complexity encountered in $\mathrm{SO}(10)$ scenarios.

(I) In the first, one-step scenario the scalar soft SUSY breaking sector, $c f$. Fig. I(left), is parametrized by the gravity induced mass parameters $m_{16}$ for the matter superfields and 


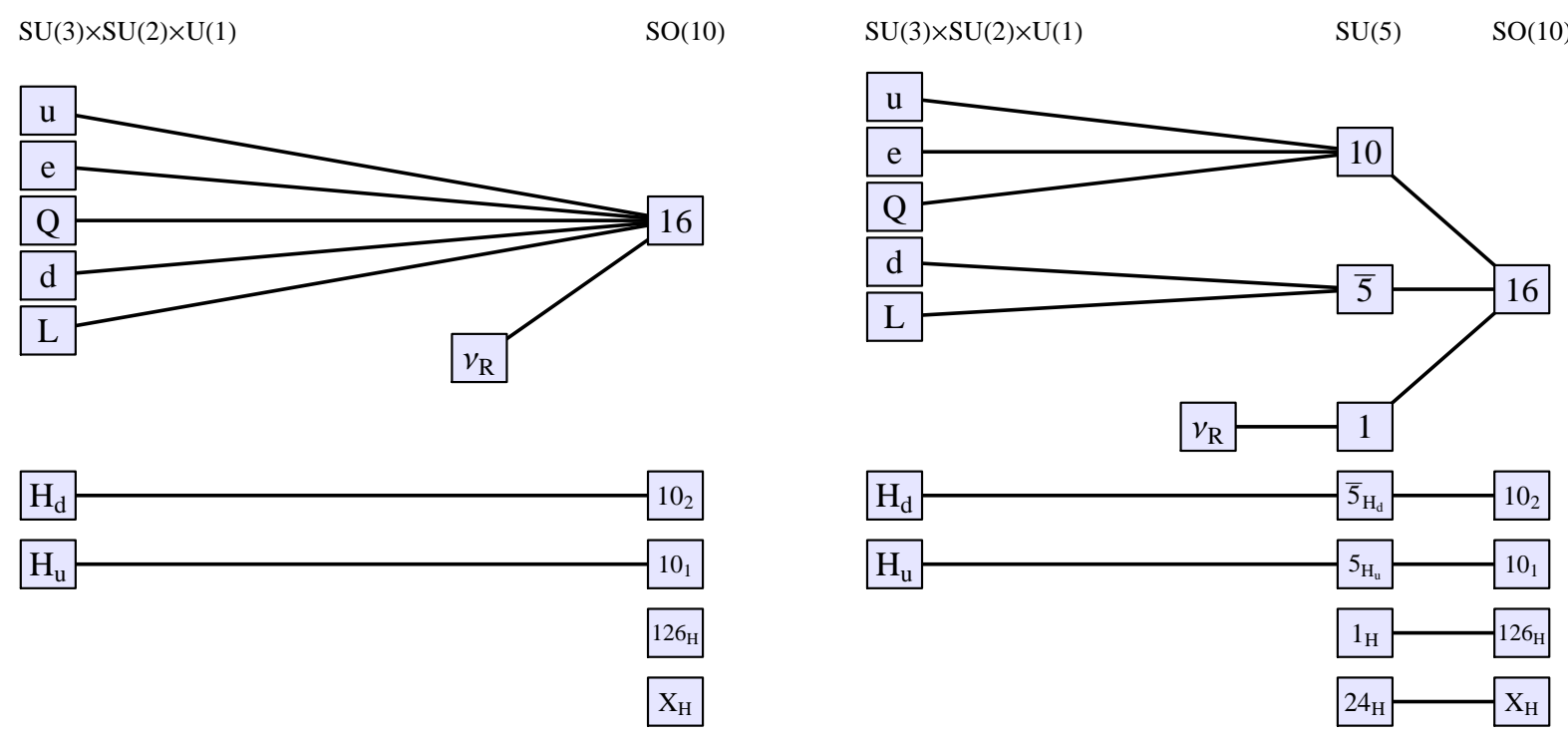

Figure 1: Systematic overview of the decomposition of the SO(10) representations, Left: one-step breaking $S O(10) \rightarrow S U(3) \times S U(2) \times U(1)$; Right: two-step breaking $S O(10) \rightarrow$ $S U(5) \rightarrow S U(3) \times S U(2) \times U(1) . X_{H}$ denotes the Higgs field that breaks $S O(10)$ while the notation of the other fields follows the general standards.

$m_{10_{1}}, m_{10_{2}}$ for two Higgs superfields at the unification scale $\Lambda_{\mathcal{U}}$. For two different Higgs fields $10_{1}$ and $10_{2}$ generating masses separately for up- and down-type fields, constraints on the range of the low-energy parameters are significantly relaxed. The scalar mass parameters are assumed universal:

$$
m_{16}=m_{10_{1}}=m_{10_{2}}=M_{0} .
$$

The GUT scale $\Lambda_{\mathcal{U}}$ is defined technically by the minimum distance of the gauge couplings within the triangle built by the running couplings near the unification point. At $\Lambda_{\mathcal{U}}$ the soft scalar mass parameters are shifted, non-universally, by D-terms associated with the SO(10) breaking to the lower-rank SM group $[6,7]$. Starting at $\Lambda_{\mathcal{U}}$, the mass parameters evolve, following the renormalization group ( $R G)$ [8], down to the electroweak scale. They define the Lagrangian parameters at the supersymmetry scale $\tilde{M}$, chosen at $1 \mathrm{TeV}$ according to the SPA convention [9]. The observed masses of sleptons and squarks, charginos/neutralinos and Higgs bosons can be expressed in terms of these Lagrangian parameters $[10,11]$. Once the masses are measured, the RG evolution from the Terascale upwards will allow us to reconstruct the physics scenario at the GUT scale $[4,12,13]$.

For the mass parameters of matter fields in the first two generations and the Higgs field $H_{d}$ the RG flow from the Terascale to the GUT scale is, effectively, not interrupted by 
any intermediate thresholds. The first two generations and the Higgs $H_{d}$ can therefore be exploited to extract the scalar GUT mass parameters $m_{16}$ and $m_{10_{1}}$, and the D-term. By contrast, the running of the mass parameters in the third generation and of the second $H_{u}$ Higgs mass parameter are affected by Yukawa interactions at the intermediate seesaw scale $[4,13,14]$. The resolution of $\nu_{R}$-higgsino and $\tilde{\nu}_{R}$-Higgs loops gives rise to kinks in the evolution of the iso-doublet scalar L-mass parameter and the $H_{u}$ Higgs mass parameter. Linking the measured $\tilde{\tau}$ and $\tilde{\nu}_{\tau}$ masses to the GUT parameter $m_{16}$, assumed universal, determines the position of the kink and thus allows us to measure the R-neutrino mass, i.e. the seesaw scale of the third generation. The analysis assumes implicitly in this approach that the problems of doublet-triplet splitting in the Higgs sector etc. are solved by mechanisms at and beyond the unification scale which do not have a significant impact on the evolution of the scalar mass parameters.

(II) In the second scenario the GUT symmetry is broken down to the Standard Model in two consecutive steps, $c f$. Fig. 1(right). At the scale $\Lambda_{\mathcal{O}}>\Lambda_{\mathcal{U}}$ the $\mathrm{SO}(10)$ symmetry breaks to $\mathrm{SU}(5)$. Apart from the D-term, the scalar mass parameter $m_{16}$ splits at $\Lambda_{\mathcal{O}}$ to three separate parameters $m_{10}, m_{\overline{5}}$ and $m_{1}$ according to the decomposition of the matter multiplet $16=10+\overline{5}+1$. At the scale $\Lambda_{\mathcal{U}}$ the $\mathrm{SU}(5)$ gauge symmetry is broken to the $\mathrm{SM}$ symmetry by a $\{24\}$ Higgs field. By restricting the heavy Higgs sector to $\{24\}$ it is assumed again that the doublet-triplet splitting problems etc. are solved by mechanisms which do not affect the evolution of the scalar mass parameters significantly. At $\Lambda_{\mathcal{U}}$ the scalar SU(5) mass parameters finally split to the MSSM parameters evolving down to the Terascale. To simplify the analysis, we assume the Higgs mass parameters $m_{10_{1}}$ and $m_{10_{2}}$ to be degenerate with $m_{16}$ at $\Lambda_{\mathcal{O}}$. Such an assumption can be motivated by string theories, in which the scales $\Lambda_{s t r}$ and $\Lambda_{\mathcal{O}}$ are identified [15]. The evolution from $\Lambda_{\mathcal{O}} \rightarrow \Lambda_{\mathcal{U}}$ depends on the field content of the high-scale $\mathrm{SU}(5)$ theory. By contrast, the evolution between $\Lambda_{\mathcal{U}}$ and the Terascale is driven by a priori experimental information, modulo the effect of the heavy R-neutrino mass parameter of the third generation. Though only a limited set of elements in the highscale scenario can be focused on, the potential of such analyses in exploring the high-scale scenario can nevertheless be elucidated. Given the multitude of potential physics scenarios near the Planck scale, these experimental projections - supplemented by other observations in the neutrino sector, proton decay, lepton flavor violating processes, and cosmological observations - will be a valuable ingredient for reconstructing the fundamental high-scale theory. 


\section{One-Step $\mathrm{SO}(10) \rightarrow$ SM Breaking}

In the $\mathrm{SO}(10)$ model which we will analyze, the matter superfields of the three generations belong to 16-dimensional representations of $\mathrm{SO}(10)$ and the standard Higgs superfields to two 10-dimensional representations, while a Higgs superfield in the 126-dimensional representation generates the Majorana masses for the right-handed neutrinos. The Yukawa couplings of matter fields $\neq \nu_{R i}$ to the 126-dimensional Higgs field are assumed to be much smaller than to the 10-plet Higgs fields. As a consequence, the Yukawa couplings in the neutrino sector coincide with the Yukawa couplings in the up-type quark matrix. Assuming the normal hierarchy for the light neutrino masses and approximate tri-bimaximal mixing, as suggested experimentally, the texture of the heavy Majorana mass matrix is predicted within the seesaw mechanism. In this framework the evolution of the soft scalar slepton mass parameters can be predicted from the unification scale down to the electroweak scale. Since the right-handed neutrino fields are neutral under the SM gauge group, they merely affect the evolution by Yukawa interactions which are sufficiently large only in the L-sector of the third generation as well as the $H_{u}$ Higgs sector.

The scalar mass parameters $m_{16}$ and the Higgs parameters $m_{10_{1}}$ and $m_{10_{2}}$ at the unification scale will be assumed universal, cf. Eq. (11). However, the breaking of the rank-5 SO(10) symmetry group to the lower rank-4 SM group generates GUT D-terms $D_{\mathcal{U}}$ such that the boundary conditions at the GUT scale finally read $[5,16]$ for the matter fields,

$$
\begin{aligned}
& m_{L}^{2}=M_{0}^{2}-3 D_{\mathcal{U}} \\
& m_{E}^{2}=M_{0}^{2}+D_{\mathcal{U}} \\
& m_{R}^{2}=M_{0}^{2}+5 D_{\mathcal{U}},
\end{aligned}
$$

and for the Higgs fields,

$$
\begin{aligned}
& m_{H_{d}}^{2}=M_{0}^{2}+2 D_{\mathcal{U}} \\
& m_{H_{u}}^{2}=M_{0}^{2}-2 D_{\mathcal{U}} .
\end{aligned}
$$

The L-isodoublet, the charged R-isosinglet, the neutral R-isosinglet and the two Higgs scalar mass parameters, are denoted by $m_{L}, m_{E}, m_{R}, m_{H_{d, u}}$, respectively. It can be shown on general grounds that the D-term is of the order of the soft SUSY breaking masses of the fields responsible for the spontaneous breaking times the gauge coupling squared [7],

$$
D_{\mathcal{U}} \sim g_{S O(10)}^{2} \mathcal{O}\left(M_{0}^{2}\right),
$$


while the detailed form depends on the specific $\mathrm{SO}(10)$ breaking mechanism. Not specifying the structure of this component of the Higgs sector, we will treat $D_{\mathcal{U}}$ as a free parameter. The evolution of the scalar masses $m_{L, E}^{2}$ from the unification scale down to the Terascale scale is determined by lepton-gaugino and slepton-gauge loops, complemented by R-neutrino-higgsino loops etc. in the third generation.

\section{a) The neutrino sector:}

Neglecting minor higher-order effects in the calculation of the Majorana neutrino mass matrix, it follows from the Higgs-\{10\} SO(10) relation

$$
Y_{\nu}=Y_{u}
$$

between the neutrino and up-type quark Yukawa matrices that

$$
Y_{\nu} \approx \operatorname{diag}\left(m_{u}, m_{c}, m_{t}\right) / v_{u}
$$

holds approximately for the neutrino Yukawa matrix; $v_{u}=v \sin \beta$, with $v$ and $\tan \beta$ being the familiar vacuum and mixing parameters in the Higgs sector. The quark masses are defined at the scale $\Lambda_{\mathcal{U}}$. Quark mixing and RG running effects in the neutrino sector are neglected in the analytical approach but properly taken into account in the numerical analysis. The effective mass matrix of the light neutrinos is constrained by the results of the neutrino oscillation experiments:

$$
m_{\nu}=U_{\mathrm{MNS}}^{*} \cdot \operatorname{diag}\left(m_{\nu_{1}}, m_{\nu_{2}}, m_{\nu_{3}}\right) \cdot U_{\mathrm{MNS}}^{\dagger} .
$$

We will assume the normal hierarchy for the light neutrino masses $m_{\nu_{i}}$, and for the MNS mixing matrix the tri-bimaximal form

$$
U_{\mathrm{MNS}}=\left(\begin{array}{ccc}
\sqrt{2 / 3} & \sqrt{1 / 3} & 0 \\
-\sqrt{1 / 6} & \sqrt{1 / 3} & \sqrt{1 / 2} \\
-\sqrt{1 / 6} & \sqrt{1 / 3} & -\sqrt{1 / 2}
\end{array}\right) .
$$

From the seesaw relation

$$
M_{\nu_{R}}=Y_{\nu} m_{\nu}^{-1} Y_{\nu}^{T} \cdot v_{u}^{2},
$$

the heavy Majorana R-neutrino mass matrix $M_{\nu_{R}}$ can finally be derived as

$$
M_{\nu_{R}} \approx \operatorname{diag}\left(m_{u}, m_{c}, m_{t}\right) m_{\nu}^{-1} \operatorname{diag}\left(m_{u}, m_{c}, m_{t}\right) .
$$




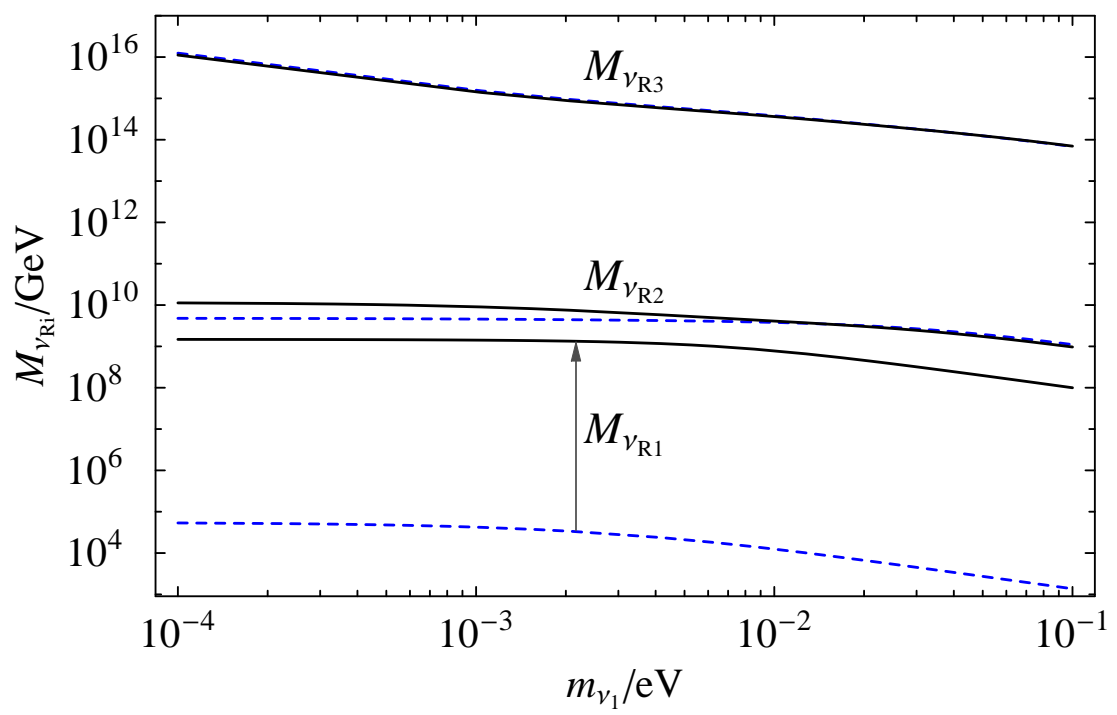

Figure 2: Masses of right-handed neutrinos $M_{\nu_{R i}}$ as functions of the lightest neutrino mass $m_{\nu_{1}}$. The dashed (blue) lines assume perfect Yukawa unification, Eq. (5). The solid (black) lines indicate the shifts of the $\nu_{R}$ masses in the first and and second generation if the Yukawa identity Eq. (5) is modified ad-hoc by a term $\kappa / v$ with $\kappa=100 \mathrm{MeV}$.

Solving Eq. (10) for the eigenvalues $M_{\nu_{R i}}(i=1,2,3)$, the heavy Majorana masses are determined by the up-quark masses $m_{u, c, t}$ at the GUT scale and the light neutrino masses $m_{\nu_{1}}, m_{\nu_{2}}, m_{\nu_{3}}$. For normal hierarchy, $m_{\nu_{3}}$ and $m_{\nu_{2}}$ are given by $m_{\nu_{3}}=\sqrt{m_{\nu_{1}}^{2}+\Delta m_{13}^{2}}$ and $m_{\nu_{2}} \approx \sqrt{m_{\nu_{1}}^{2}+\Delta m_{12}^{2}}$, with the mass squared differences $\Delta m_{13}^{2} \approx 2 \cdot 10^{-3} \mathrm{eV}^{2}$ and $\Delta m_{12}^{2} \approx 8 \cdot 10^{-5} \mathrm{eV}^{2}$, measured in neutrino oscillation experiments. The $M_{\nu_{R i}}$ spectrum is then predicted as a function of the masses of the light neutrinos. Quite generally, the solution for the eigenvalues can be approximated to a high level of accuracy by the relations [see also Ref. [17]]:

$$
\begin{aligned}
M_{\nu_{R 1}} & \approx \frac{m_{\nu_{1}}+2 m_{\nu_{2}}+3 m_{\nu_{3}}}{3 m_{\nu_{1}} m_{\nu_{2}}+2 m_{\nu_{1}} m_{\nu_{3}}+m_{\nu_{2}} m_{\nu_{3}}} m_{u}^{2} \\
M_{\nu_{R 2}} & \approx \frac{4 m_{\nu_{1}}+2 m_{\nu_{2}}+0 m_{\nu_{3}}}{3 m_{\nu_{1}} m_{\nu_{2}}+2 m_{\nu_{1}} m_{\nu_{3}}+m_{\nu_{2}} m_{\nu_{3}}} m_{c}^{2} \\
M_{\nu_{R 3}} & \approx \frac{3 m_{\nu_{1}} m_{\nu_{2}}+2 m_{\nu_{1}} m_{\nu_{3}}+m_{\nu_{2}} m_{\nu_{3}}}{6 m_{\nu_{1}} m_{\nu_{2}} m_{\nu_{3}}} m_{t}^{2} .
\end{aligned}
$$

Thus the mass spectrum of the R-neutrinos is strongly ordered in $\mathrm{SO}(10)$ with minimal Higgs content, $M_{\nu_{R 3}}: M_{\nu_{R 2}}: M_{\nu_{R 1}} \sim m_{t}^{2}: m_{c}^{2}: m_{u}^{2}$.

The numerical evaluation, including refinements like RG running effects, is displayed in Fig. 2 for a wide range of $m_{\nu_{1}}$ values. The analytical approximation, Eq. (11), is very 
accurate across the entire range, from small $m_{\nu_{1}}$ with strong ordering of the hierarchical light neutrino masses, $m_{\nu_{1}} \ll m_{\nu_{2}} \ll m_{\nu_{3}}$, up to nearly degenerate light neutrino masses, $m_{\nu_{i}} \rightarrow m_{\nu_{1}}$. The heavy R-neutrino masses read

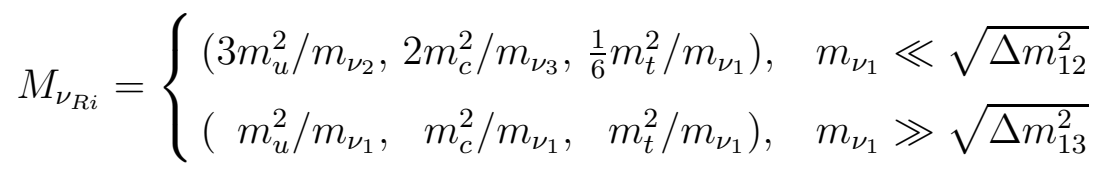

in these two limits.

It should be noted that the prediction for the third-generation R-neutrino mass $M_{\nu_{R 3}}$ is quite robust, contrary to the second and the first generation in particular. Modifying the relation between the neutrino and up-type quark Yukawa couplings, Eqs. (5), ad-hoc by a small additional term, $Y_{\nu}=Y_{u}+\kappa / v$, with $\kappa \sim$ a few hundred $\mathrm{MeV}$ associated potentially with a more complex Higgs scenario, Planck-scale suppressed contributions or non-perturbative effects at small mass scales, the first generation R-neutrino mass is lifted to $\sim 10^{9} \mathrm{GeV}$. This just illustrates that a small modification of the $\nu$-up quark Yukawa identity is sufficient to reconcile the mass estimate with limits suggested within leptogenesis scenarios for the matter-antimatter asymmetry in the Universe [18].

The small mixing of the right-handed neutrinos,

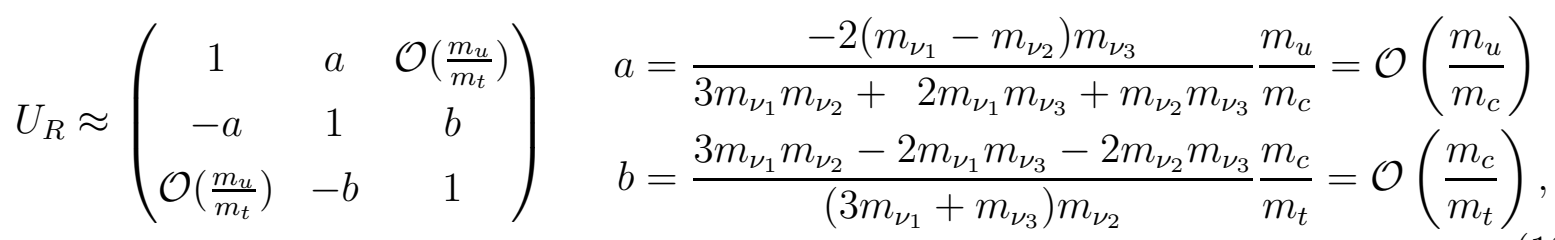

hardly affects the analysis, since it enters only indirectly into the RG evolution of the slepton mass parameters when the right-handed neutrinos are decoupled at their own mass scale.

The Yukawa mass matrix squared, which determines the connection of the slepton masses in the third generation at low and high scales, is dominated by the 33 element,

$$
\left(Y_{\nu}^{\dagger} Y_{\nu}\right)_{33} \approx m_{t}^{2}\left(\Lambda_{\mathcal{U}}\right) / v_{u}^{2} \approx 0.3
$$

while the other elements are suppressed to a level of $10^{-2}$ down to $10^{-5}$. 


\section{b) Scalar mass parameters:}

The slepton systems of the first two generations depend, to leading order, on four parameters: the two fundamental scalar and gaugino mass parameters, the D-term and the unification scale. They can be uniquely determined from the unification scale of the gauge couplings, the L- and R-slepton masses and the masses of the charginos and neutralinos. The complementary analysis of squarks provides an independent cross-check of the underlying picture.

Analytic relations, for the sake of clarity, are presented only to leading logarithmic order while the subsequent numerical analyses include the non-logarithmic higher order contributions. To leading order, the solutions of the RG equations, the masses of the scalar selectrons and the L-type $e$-sneutrino, can be expressed in terms of the high scale parameter $m_{16}=M_{0}$, the universal gaugino mass parameter $M_{1 / 2}$ and the GUT and electroweak D-terms, $D_{\mathcal{U}}$ and $D_{E W}=M_{Z}^{2} / 2 \cos 2 \beta$, respectively:

$$
\begin{aligned}
m_{\tilde{e}_{R}}^{2} & =M_{0}^{2}+D_{\mathcal{U}}+\alpha_{R} M_{1 / 2}^{2}-\frac{6}{5} S^{\prime}-2 s_{W}^{2} D_{E W} \\
m_{\tilde{e}_{L}}^{2} & =M_{0}^{2}-3 D_{\mathcal{U}}+\alpha_{L} M_{1 / 2}^{2}+\frac{3}{5} S^{\prime}-c_{2 W} D_{E W} \\
m_{\tilde{\nu}_{e L}}^{2} & =M_{0}^{2}-3 D_{\mathcal{U}}+\alpha_{L} M_{1 / 2}^{2}+\frac{3}{5} S^{\prime}+D_{E W}
\end{aligned}
$$

[as usual, $s_{W}^{2}=\sin ^{2} \theta_{W}$ etc.]. The coefficients $\alpha_{L}$ and $\alpha_{R}$ are given by the gaugino/gauge boson loops in the RG evolution from the global supersymmetry scale $\tilde{M}[9]$ to the unification scale $\Lambda_{\mathcal{U}}$

$$
\begin{aligned}
\alpha_{L} & =\frac{3}{10} f_{1}+\frac{3}{2} f_{2} \\
\alpha_{R} & =\frac{6}{5} f_{1} \\
f_{i} & =\frac{1}{b_{i}}\left(1-\left[1+\frac{\alpha_{\mathcal{U}}}{4 \pi} b_{i} \log \frac{\Lambda_{\mathcal{U}}^{2}}{\tilde{M}^{2}}\right]^{-2}\right) \text { with }\left(b_{1}, b_{2}\right)=\left(\frac{33}{5}, 1\right),
\end{aligned}
$$

and the numerical evaluation finally yields $\alpha_{R} \approx 0.15$ and $\alpha_{L} \approx 0.5$ for $\tilde{M}=1 \mathrm{TeV}$. The universal gaugino mass parameter $M_{1 / 2}$ can be pre-determined in the chargino/neutralino sector. The non-universal initial conditions in the evolution due to the D-terms generate the small generation-independent corrections

$$
S^{\prime}=-4 D_{\mathcal{U}} \frac{\alpha_{1}(\tilde{M})}{\alpha_{1}\left(\Lambda_{\mathcal{U}}\right)}
$$

cf. Ref. [16]. 
The Higgs parameter $m_{10_{1}}=M_{0}$ can be calculated analogously,

$$
m_{H_{d}}^{2}=M_{0}^{2}+2 D_{\mathcal{U}}+\alpha_{L} M_{1 / 2}^{2}+\frac{3}{5} S^{\prime}-\Delta_{\tau}-3 \Delta_{b} .
$$

$\Delta_{\tau}$ and $\Delta_{b}$ describe contributions involving $\tau$ and $b$ loops, respectively, with correspondingly large Yukawa couplings, see Eqs. (21) and (23) below.

The scalar masses of the third generation receive additional contributions from $\nu_{R \tau}$-higgsino loops etc., coupled by Yukawa interactions with the L and $\mathrm{R}$ fields. The masses of the third generation are shifted relative to the masses of the first two generations by two terms $[4,13]$ :

$$
\begin{aligned}
m_{\tilde{\tau}_{R}}^{2} & =m_{\tilde{e}_{R}}^{2}+m_{\tau}^{2}-2 \Delta_{\tau} \\
m_{\tilde{\tau}_{L}}^{2} & =m_{\tilde{e}_{L}}^{2}+m_{\tau}^{2}-\Delta_{\tau}-\Delta_{\nu_{\tau}} \\
m_{\tilde{\nu}_{\tau L}}^{2} & =m_{\tilde{\nu}_{e L}}^{2}-\Delta_{\tau}-\Delta_{\nu_{\tau}} .
\end{aligned}
$$

The Higgs parameter $m_{H_{u}}^{2}$ is given analogously, in the universality class $m_{10_{2}}=M_{0}$, by

$$
m_{H_{u}}^{2}=M_{0}^{2}-2 D_{\mathcal{U}}-\frac{3}{5} S^{\prime}+\alpha_{L} M_{1 / 2}^{2}-\Delta_{\nu_{\tau}}-3 \Delta_{t} .
$$

The shifts $\Delta_{\tau}$ and $\Delta_{\nu_{\tau}}$, generated by loops [19] involving charged lepton and neutrino superfields, respectively, are predicted by the renormalization group in the $\mathrm{SO}(10)$ scenario,

$$
\begin{aligned}
\Delta_{\tau} & \approx \frac{m_{\tau}^{2}\left(\Lambda_{\mathcal{U}}\right)}{8 \pi^{2} v_{d}^{2}}\left(3 M_{0}^{2}+A_{0}^{2}\right) \log \frac{\Lambda_{\mathcal{U}}^{2}}{\tilde{M}^{2}} \\
\Delta_{\nu_{\tau}} & \approx \frac{m_{t}^{2}\left(\Lambda_{\mathcal{U}}\right)}{8 \pi^{2} v_{u}^{2}}\left(3 M_{0}^{2}+A_{0}^{2}\right) \log \frac{\Lambda_{\mathcal{U}}^{2}}{M_{\nu_{R 3}}^{2}},
\end{aligned}
$$

including the [universal] trilinear coupling $A_{0}$, while

$$
\begin{aligned}
\Delta_{b} & \approx \frac{m_{b}^{2}\left(\Lambda_{\mathcal{U}}\right)}{8 \pi^{2} v_{d}^{2}}\left(3 M_{0}^{2}+A_{0}^{2}\right) \log \frac{\Lambda_{\mathcal{U}}^{2}}{\tilde{M}^{2}} \\
\Delta_{t} & \approx \frac{m_{t}^{2}\left(\Lambda_{\mathcal{U}}\right)}{8 \pi^{2} v_{u}^{2}}\left(3 M_{0}^{2}+A_{0}^{2}\right) \log \frac{\Lambda_{\mathcal{U}}^{2}}{\tilde{M}^{2}}
\end{aligned}
$$

account similarly for the running from the Tera- to the GUT scale induced by $b, t$ loops. The parameter $m_{t}\left(\Lambda_{\mathcal{U}}\right)=m_{t}(v)\left(\Lambda_{\mathcal{U}} / v\right)^{\gamma}$ is the running top quark mass with, approximately, $\gamma=\left(6 y_{t}^{2}-16 / 3 g_{s}^{2}\right) /\left(16 \pi^{2}\right)$, the top-quark Yukawa coupling $y_{t}$ and the QCD coupling $g_{s}$ evaluated at the electroweak scale $v ; v_{d(u)}=v \cos \beta(v \sin \beta)$.

While the slepton masses, specifically the sum $m_{\tilde{\tau}_{L}}^{2}+m_{\tilde{\tau}_{R}}^{2}=m_{\tilde{\tau}_{1}}^{2}+m_{\tilde{\tau}_{2}}^{2}$ of the third generation, are experimentally accessible directly, the Higgs mass parameters,

$$
m_{H_{d(u)}}^{2}=M_{A}^{2} \sin ^{2} \beta \cos ^{2} \beta-|\mu|^{2} \mp \frac{1}{2} M_{Z}^{2} \cos 2 \beta
$$




\begin{tabular}{|l|l|l||l|c|l|}
\hline Parameter & Value & Error & Parameter & Value & Error \\
\hline \hline$m_{\tilde{e}_{R}}$ & $140.9 \mathrm{GeV}$ & $0.05 \mathrm{GeV}$ & $\mu$ & $481.1 \mathrm{GeV}$ & $4.5 \mathrm{GeV}$ \\
$m_{\tilde{e}_{L}}$ & $190.4 \mathrm{GeV}$ & $0.4 \mathrm{GeV}$ & $\tan \beta$ & 10.0 & 1.0 \\
$m_{\tilde{\nu}_{e}}$ & $173.4 \mathrm{GeV}$ & $1.3 \mathrm{GeV}$ & $M_{1 / 2}$ & $250.0 \mathrm{GeV}$ & $0.4 \mathrm{GeV}$ \\
$m_{\tilde{\tau}_{1}}$ & $104.2 \mathrm{GeV}$ & $0.3 \mathrm{GeV}$ & $A_{0}$ & $-640.0 \mathrm{GeV}$ & $13 \mathrm{GeV}$ \\
$m_{\tilde{\tau}_{2}}$ & $187.8 \mathrm{GeV}$ & $1.1 \mathrm{GeV}$ & & & \\
$m_{\tilde{\nu}_{\tau}}$ & $154.7 \mathrm{GeV}$ & $1.6 \mathrm{GeV}$ & & & \\
\hline
\end{tabular}

Table 1: Expected experimental errors for the determination of sfermion masses and other underlying parameters in the sample one-step $S O(10)$ scenario. [The errors quoted correspond to $1 \sigma$.

can be determined from the pseudo-scalar Higgs-boson mass $M_{A}$ and the higgsino mass parameter $\mu$.

\section{c) Numerical results:}

Anticipating high-precision measurements at future colliders, such an $\mathrm{SO}(10)$ scenario can be investigated in central facets. As a concrete example, we study the following LR-extended scenario with MSSM parameters close to SPS1a/a' [9, 20]:

$$
\begin{aligned}
M_{0} & =90 \mathrm{GeV} & \tan \beta & =10 \\
M_{1 / 2} & =250 \mathrm{GeV} & \operatorname{sgn}(\mu) & =+ \\
A_{0} & =-640 \mathrm{GeV} & &
\end{aligned}
$$

and

$$
\begin{aligned}
D_{\mathcal{U}} & =-0.9 \cdot 10^{3} \mathrm{GeV}^{2} \\
M_{\nu_{R 3}} & =7.2 \cdot 10^{14} \mathrm{GeV}
\end{aligned}
$$

The low-energy and cosmological data of this scenario are compatible with observations: QCD coupling $\alpha_{s}\left(M_{Z}\right)=0.119$, electroweak mixing parameter $\sin ^{2} \theta_{\text {eff }}^{\text {lept }}=0.23140$, radiative $b$ decay $\operatorname{BR}(b \rightarrow s \gamma)=3.32 \cdot 10^{-4}$, deviation of the muon anomalous magnetic moment from SM value $\Delta a_{\mu}=\Delta\left(g_{\mu}-2\right) / 2=15.3 \cdot 10^{-10}$, and cold-dark-matter density $\Omega h_{0}^{2}=0.110$.

The estimated experimental precision for the measurements of slepton, sneutrino and gaugino masses at LHC and ILC can be extrapolated from the results of Refs. [4,9,21-25], which are based on detailed simulations. The masses of the charged sleptons can be measured with high precision in slepton pair production at ILC [21], while the sneutrino masses can 

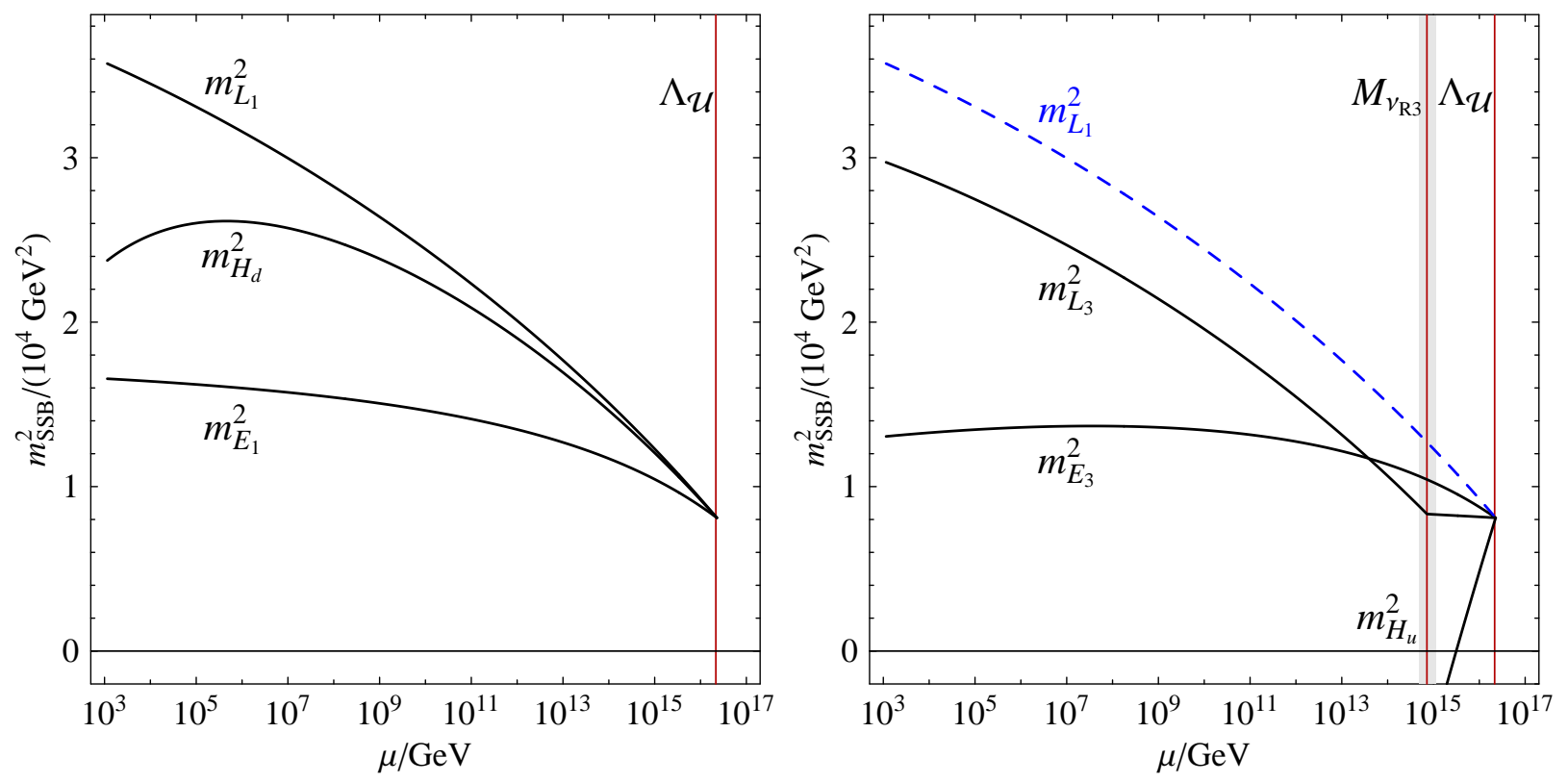

Figure 3: Left: Evolution of the L,R slepton mass parameters of the first generation for one-step breaking with the D-term, merely for illustration, set to zero. Right: Evolution of the first and third generation $L, R$ slepton and Higgs mass parameters $\left[D_{\mathcal{U}}=0\right]$ for one-step breaking; the kink in $m_{L_{3}}^{2}$ is generated by the right-handed neutrino with mass $M_{\nu_{R 3}}$ close to $10^{15} \mathrm{GeV}$.

be determined accurately from the decays of charginos [4]. The Higgs masses in Eq. (25) are expressed in terms of the parameters $\mu$ and $\tan \beta$, which can be derived accurately from the analysis of chargino and neutralino observables [22,23]. The pseudoscalar Higgs mass $M_{A}$, on the other hand, can be measured in associated Higgs pair production [26]. The direct determination of the $A$ parameters is difficult, $c f$. Refs. [27-29]. However, taking into account stop/sbottom mass measurements but leaving out the sleptons of the third generation and the Higgs bosons to bypass the (yet to be determined) parameters of the right-handed neutrinos, a global analysis including loop effects 11 leads to an accurate determination of $A_{0}$ at the level of $2 \%$ [9,25]. The relevant parameters are summarized in Tab. 1. including the estimated experimental errors.

The measurement of the slepton and sneutrino masses of the first two generations allows

\footnotetext{
${ }^{1}$ Based on this reduced set of observables, errors on $A_{0}$ of about $6 \mathrm{GeV}$ have been predicted for SPS1a and SPS1a ${ }^{\prime}$ with $A_{0}$ values of -100 and $-300 \mathrm{GeV}$, respectively. Fixing the error on $A_{0}$ to $2 \%$, i.e. $12 \mathrm{GeV}$, for the present benchmark point with large $A_{0}=-640 \mathrm{GeV}$ can therefore be considered as a conservative estimate. Thanks go to P. Bechtle and D. Zerwas for running Fittino and Sfitter, respectively, for estimating the increase of the error on $A_{0}$ in SPS1a and SPS1a' for the reduced set of experimental observables.
} 


\begin{tabular}{|l|c|c|}
\hline Parameters in $\mathrm{SO}(10) \rightarrow \mathrm{SM}$ & Ideal & Error \\
\hline \hline unification scale $\Lambda_{\mathcal{U}}$ & $2.16 \cdot 10^{16} \mathrm{GeV}$ & $0.02 \cdot 10^{16} \mathrm{GeV}$ \\
matter scalar mass $M_{0}$ & $90 \mathrm{GeV}$ & $0.25 \mathrm{GeV}$ \\
GUT D-term $\sqrt{-D_{\mathcal{U}}}$ & $30 \mathrm{GeV}$ & $0.9 \mathrm{GeV}$ \\
\hline heaviest R-neutrino mass $M_{\nu_{R 3}}$ & $7.2 \cdot 10^{14} \mathrm{GeV}$ & {$[4.8,11] \cdot 10^{14} \mathrm{GeV}$} \\
lightest neutrino mass $m_{\nu_{1}}$ & $3.5 \cdot 10^{-3} \mathrm{eV}$ & {$[1.6,6.7] \cdot 10^{-3} \mathrm{eV}$} \\
\hline
\end{tabular}

Table 2: Reconstruction of high-scale $S O(10)$ parameters in one-step $S O(10) \rightarrow S M$ breaking, and masses of the heavy 3-generation $R$-neutrino and the lightest neutrino [The errors quoted correspond to $1 \sigma]$.

us to extract the common sfermion parameter $m_{16}=M_{0}$ as well as the D-term $D_{\mathcal{U}}$. The relations are given in Eq. (15) in leading logarithmic approximation for the RG running. Including the complete one-loop and the leading two-loop corrections, the evolution of the scalar mass parameters, $\left(m_{L 1}^{2}, m_{E 1}^{2}\right)$, is displayed in Fig. 3 (left) for the first two generations. The curves describe the evolution of the universal $L$ and $R$ mass parameters with, merely for illustration, the D-term set to zero.

With the estimated errors in Tab. 1, the high-scale parameters can be calculated, by combining the slepton and Higgs sectors, as shown in Tab. 2. The RG evolution equations are evaluated to 2-loop order by means of the SPheno program [30]. The table indicates that the high-scale parameters $M_{0}$ and $D_{\mathcal{U}}$, driven by the slepton analysis, can be reconstructed at per-mill to per-cent accuracy.

The right-handed neutrino affects the evolution of the L mass parameter $m_{L 3}^{2}$ in the third generation and the Higgs parameter $m_{H_{u}}^{2}$. [Note that $m_{H_{u}}^{2}+\mu^{2}$ turns negative only at the small scale $Q \simeq 350 \mathrm{GeV}$.] The characteristic difference in the evolution between $m_{L 3}^{2}$ and $m_{L 1}^{2}$ is exemplified in Fig. 33(right) for a right-handed neutrino mass $M_{\nu_{R 3}}$ of $7.2 \cdot 10^{14} \mathrm{GeV}$. From the universality point $\Lambda_{\mathcal{U}}$, where $m_{L_{3}}$ and $m_{L_{1}}$ are equal, down to the kink at $M_{\nu_{R 3}}$ the evolution of $m_{L_{3}}$ is affected by the right-handed neutrino, $m_{L 1}$ however is not. Below the kink the difference between $m_{L_{3}}$ and $m_{L_{1}}$ is reduced to the standard loop correction $\Delta_{\tau}$ and the small $\tau$ mass. The position of the kink at $M_{\nu_{R 3}}$ can be derived from the measured slepton and sneutrino masses if the scalar mass parameters are universal at the unification scale. Neglecting the small term $m_{\tau}^{2}$, the right-handed neutrino mass is fixed by the intersection of the parameter $\Delta_{\nu_{\tau}}$ as a function of $M_{\nu_{R 3}}$ with the measured value extracted from the 


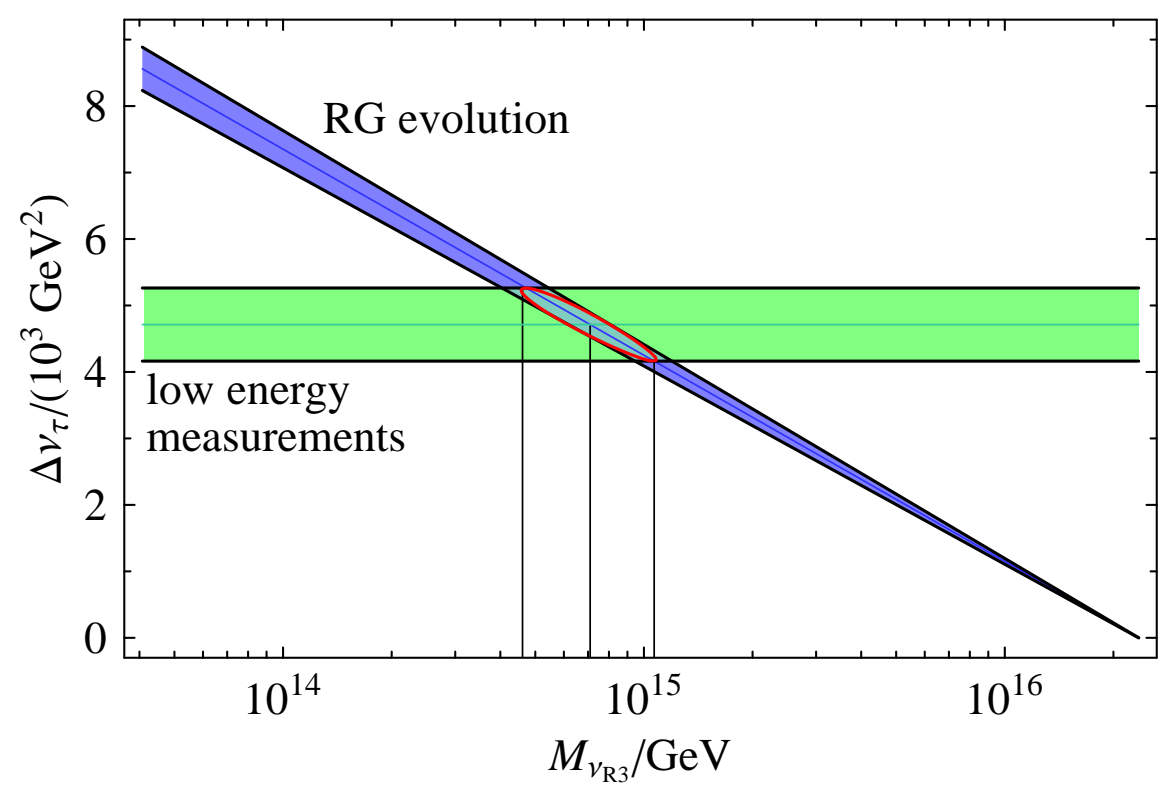

Figure 4: Shift $\Delta_{\nu_{\tau}}$ of the third generation L slepton mass parameter generated by loops involving heavy neutrino R-superfields. The blue wedge corresponds to the prediction Eq. (22) of the renormalization group $[R G]$, whereas the green band is determined by low-energy mass measurements.

slepton masses:

$$
\begin{aligned}
\Delta_{\nu_{\tau}} & =\frac{1}{2}\left[m_{\tilde{e}_{L}}^{2}+m_{\tilde{e}_{R}}^{2}-3 m_{\tilde{\nu}_{e L}}^{2}\right]-\frac{1}{2}\left[m_{\tilde{\tau}_{1}}^{2}+m_{\tilde{\tau}_{2}}^{2}-3 m_{\tilde{\nu}_{\tau L}}^{2}\right] \\
& =\frac{m_{t}^{2}\left(\Lambda_{\mathcal{U}}\right)}{8 \pi^{2} v_{u}^{2}}\left(3 M_{0}^{2}+A_{0}^{2}\right) \log \frac{\Lambda_{\mathcal{U}}^{2}}{M_{\nu_{R 3}}^{2}}
\end{aligned}
$$

In practice the separate evaluation of the charged slepton and sneutrino mass differences,

$$
\begin{aligned}
\Delta_{\nu_{\tau}} & =m_{\tilde{\nu}_{e L}}^{2}-m_{\tilde{\nu}_{\tau} L}^{2}-\Delta_{\tau} \\
& =m_{\tilde{e}_{L}}^{2}+m_{\tilde{e}_{R}}^{2}-m_{\tilde{\tau}_{L}}^{2}-m_{\tilde{\tau}_{R}}^{2}-3 \Delta_{\tau}
\end{aligned}
$$

proves useful to obtain a more precise value, $\Delta_{\nu_{\tau}}^{e x p}=(4.7 \pm 0.5) \cdot 10^{3} \mathrm{GeV}^{2}$. As a result, for the parameters of the LR extended point SPS1a' introduced earlier, the right-handed neutrino mass of the third generation is estimated in the margin

$$
M_{\nu R_{3}}=10^{14.86 \pm 0.19} \mathrm{GeV}=7.2_{-2.5}^{+4.0} \cdot 10^{14} \mathrm{GeV}
$$

as evident from Fig. 4 . Thus, the effect of the heavy $\nu_{R 3}$ mass can indeed be traced back from measured slepton masses in universal supersymmetric theories. 
Based on this estimate of $M_{\nu R_{3}}$, the seesaw mechanism determines the value of the lightest neutrino mass ( $c f$. Fig. 2) to

$$
m_{\nu_{1}}=10^{-2.51 \pm 0.32} \mathrm{eV}=3.1_{-1.5}^{+3.5} \cdot 10^{-3} \mathrm{eV} .
$$

The second lightest neutrino mass $m_{\nu_{2}} \simeq(1.0 \pm 0.1) \cdot 10^{-2} \mathrm{eV}$ is about three times larger

while the third neutrino mass $m_{\nu_{3}} \simeq 5 \cdot 10^{-2} \mathrm{eV}$ coincides with the mass difference $\Delta m_{13}$ measured in the neutrino oscillation experiments.

Thus, the combination of $\mathrm{SO}(10)$ symmetry and seesaw mechanism leads, besides the highscale universal SUSY parameters, to the determination of the heavy Majorana mass $M_{\nu_{R 3}}$ of the third generation and, in a consecutive step, to the value of the lightest neutrino mass $m_{\nu_{1}}$ in hierarchical theories. This conclusion is valid for scenarios in which threshold effects at $\Lambda_{\mathcal{U}}$ and the mechanisms for solving the doublet-triplet splitting problem in the Higgs sector etc. do not have a significant impact on the evolution of the scalar mass parameters. The analysis could be adjusted correspondingly if the structure of the high-scale scenario demanded a more complex extension.

\section{Two-Step $\mathrm{SO}(10) \rightarrow \mathrm{SU}(5) \rightarrow$ SM Breaking}

If the grand unification symmetry $\mathrm{SO}(10)$ is broken in two steps down to the Standard Model, cf. Fig. 1(right), the evolution between the Terascale and the $\mathrm{SU}(5)$ scale $\Lambda_{\mathcal{U}}$ is determined by measured parameters: gauge couplings, scalar mass parameters and neutrino parameters. However, the evolution between the $\mathrm{SU}(5)$ scale $\Lambda_{\mathcal{U}}$ and the $\mathrm{SO}(10)$ scale $\Lambda_{\mathcal{O}}$ depends on the high-scale physics scenario, comprising new matter, gauge and Higgs fields, and potentially effective elements of gravity interactions.

In the present approach we restrict these degrees of freedom to the $\{24\}$ Higgs field which breaks the SU(5) gauge symmetry. Other degrees of freedom and/or additional mechanisms generating the doublet-triplet splitting and prolonging the proton lifetime are assumed to be weakly coupled to the scalar mass parameters below $\Lambda_{\mathcal{O}}$. This assumption would fail if these problems would be solved by Higgs fields in large representations; in this case the analysis would be modified significantly. The two-step analysis is therefore much less robust than the previous one-step analysis. Nevertheless it can serve as an illustration for the potential of parametric analyses if the fundamental structure of a more complex scenario is theoretically pre-determined. 
a) Evolution Terascale $\rightarrow \Lambda_{\mathcal{U}}$

Based on the standard SU(5) decomposition, the scalar masses evolve from the electroweak scale to the $\mathrm{SU}(5)$ scale $\Lambda_{\mathcal{U}}$ according to the rules

$$
\begin{aligned}
m_{\tilde{e}_{R}}^{2} & =m_{10,1}^{2}+\alpha_{R} M_{\mathcal{U}, 1 / 2}^{2}-\frac{6}{5} S^{\prime}-2 s_{W}^{2} D_{E W} \\
m_{\tilde{e}_{L}}^{2} & =m_{5,1}^{2}+\alpha_{L} M_{\mathcal{U}, 1 / 2}^{2}+\frac{3}{5} S^{\prime}-c_{2 W} D_{E W} \\
m_{\tilde{\nu}_{e L}}^{2} & =m_{5,1}^{2}+\alpha_{L} M_{\mathcal{U}, 1 / 2}^{2}+\frac{3}{5} S^{\prime}+D_{E W}
\end{aligned}
$$

for the matter fields of the first two generations while the masses of the third generation,

$$
\begin{aligned}
m_{\tilde{\tau}_{R}}^{2} & =m_{10,3}^{2}+\alpha_{R} M_{\mathcal{U}, 1 / 2}^{2}-\frac{6}{5} S^{\prime}-2 s_{W}^{2} D_{E W}+m_{\tau}^{2}-2 \Delta_{\tau} \\
m_{\tilde{\tau}_{L}}^{2} & =m_{5,3}^{2}+\alpha_{L} M_{\mathcal{U}, 1 / 2}^{2}+\frac{3}{5} S^{\prime}-c_{2 W} D_{E W}+m_{\tau}^{2}-\Delta_{\tau}-\Delta_{\nu_{\tau}} \\
m_{\tilde{\nu}_{\tau L}}^{2} & =m_{5,3}^{2}+\alpha_{L} M_{\mathcal{U}, 1 / 2}^{2}+\frac{3}{5} S^{\prime}+D_{E W}-\Delta_{\tau}-\Delta_{\nu_{\tau}}
\end{aligned}
$$

are affected in addition by the Yukawa interactions in the same way as Eqs. (21|222). The indices 1,3 next to the $\mathrm{SU}(5)$ multiplet characteristics denote the generation numbers. The evolution of the Higgs mass parameters read correspondingly

$$
\begin{aligned}
& m_{H_{d}}^{2}=m_{5_{1}}^{2}+\alpha_{L} M_{\mathcal{U}, 1 / 2}^{2}+\frac{3}{5} S^{\prime}-\Delta_{\tau}-3 \Delta_{b} \\
& m_{H_{u}}^{2}=m_{5_{2}}^{2}+\alpha_{L} M_{\mathcal{U}, 1 / 2}^{2}-\frac{3}{5} S^{\prime}-\Delta_{\nu_{\tau}}-3 \Delta_{t} .
\end{aligned}
$$

Since $\mathrm{SU}(5)$ and $\mathrm{SU}(3) \times \mathrm{SU}(2) \times \mathrm{U}(1)$ are both rank-4 symmetry groups, no GUT D-term is induced in this symmetry breaking step.

The $\Delta$ 's are given by the loop corrections,

$$
\begin{aligned}
\Delta_{\tau} & =\frac{m_{\tau}^{2}\left(\Lambda_{\mathcal{U}}\right)}{8 \pi^{2} v_{d}^{2}}\left(m_{10,3}^{2}+m_{5,3}^{2}+m_{5_{1}}^{2}+A_{5,3}^{2}\right) \log \frac{\Lambda_{\mathcal{U}}^{2}}{\tilde{M}^{2}} \\
\Delta_{\nu_{\tau}} & =\frac{m_{t}^{2}\left(\Lambda_{\mathcal{U}}\right)}{8 \pi^{2} v_{u}^{2}}\left(m_{1,3}^{2}+m_{5,3}^{2}+m_{5_{2}}^{2}+A_{1,3}^{2}\right) \log \frac{\Lambda_{\mathcal{U}}^{2}}{M_{\nu_{R 3}}^{2}}
\end{aligned}
$$

and

$$
\begin{aligned}
\Delta_{b} & =\frac{m_{b}^{2}\left(\Lambda_{\mathcal{U}}\right)}{8 \pi^{2} v_{d}^{2}}\left(m_{10,3}^{2}+m_{5,3}^{2}+m_{5_{1}}^{2}+A_{5,3}^{2}\right) \log \frac{\Lambda_{\mathcal{U}}^{2}}{\tilde{M}^{2}} \\
\Delta_{t} & =\frac{m_{t}^{2}\left(\Lambda_{\mathcal{U}}\right)}{8 \pi^{2} v_{u}^{2}}\left(2 m_{10,3}^{2}+m_{5_{2}}^{2}+A_{10,3}^{2}\right) \log \frac{\Lambda_{\mathcal{U}}^{2}}{\tilde{M}^{2}},
\end{aligned}
$$


while the contributions to the $S^{\prime}$ parameter trace back to the non-universal Higgs mass parameters at the scale $\Lambda_{\mathcal{U}}$,

$$
S^{\prime}=\frac{\alpha_{1}(\tilde{M})}{\alpha_{1}\left(\Lambda_{\mathcal{U}}\right)}\left(m_{5_{2}}^{2}-m_{\overline{5}_{1}}^{2}\right)
$$

$M_{\mathcal{U}, 1 / 2}$ is the universal gaugino mass parameter at the $\mathrm{SU}(5)$ scale $\Lambda_{\mathcal{U}}$.

b) Evolution $\Lambda_{\mathcal{U}} \rightarrow \Lambda_{\mathcal{O}}$ :

The subsequent evolution from the $\mathrm{SU}(5)$ breaking scale $\Lambda_{\mathcal{U}}$ to the $\mathrm{SO}(10)$ breaking scale $\Lambda_{\mathcal{O}}$ unifies the mass parameters $m_{10}, m_{\overline{5}}$ and $m_{1}$ to $m_{16}$,

$$
\begin{aligned}
m_{10,1}^{2} & =M_{0}^{2}+D_{\mathcal{O}}+\alpha_{R}^{\prime} M_{1 / 2}^{2} \\
m_{\overline{5}, 1}^{2} & =M_{0}^{2}-3 D_{\mathcal{O}}+\alpha_{L}^{\prime} M_{1 / 2}^{2}
\end{aligned}
$$

and

$$
\begin{aligned}
m_{10,3}^{2} & =M_{0}^{2}+D_{\mathcal{O}}+\alpha_{R}^{\prime} M_{1 / 2}^{2}-3 \Delta_{t}^{\prime}-2 \Delta_{b}^{\prime} \\
m_{5,3}^{2} & =M_{0}^{2}-3 D_{\mathcal{O}}+\alpha_{L}^{\prime} M_{1 / 2}^{2}-\Delta_{\nu_{\tau}}^{\prime}-4 \Delta_{b}^{\prime} \\
m_{1,3}^{2} & =M_{0}^{2}+5 D_{\mathcal{O}}-5 \Delta_{\nu_{\tau}}^{\prime} .
\end{aligned}
$$

The Higgs parameters transform according to

$$
\begin{aligned}
& m_{\overline{5}_{1}}^{2}=M_{0}^{2}+2 D_{\mathcal{O}}+\alpha_{L}^{\prime} M_{1 / 2}^{2}-4 \Delta_{b}^{\prime}-\frac{24}{5} \Delta_{\lambda}^{\prime} \\
& m_{5_{2}}^{2}=M_{0}^{2}-2 D_{\mathcal{O}}+\alpha_{L}^{\prime} M_{1 / 2}^{2}-\Delta_{\nu_{\tau}}^{\prime}-3 \Delta_{t}^{\prime}-\frac{24}{5} \Delta_{\lambda}^{\prime}
\end{aligned}
$$

The D-term associated with the breaking of the rank-5 SO(10) to the rank-4 SU(5) symmetry is given again by a relation analogous to Eq. (44) for a Higgs- $\{16\}$ field, for example, responsible for the symmetry breaking of $\mathrm{SO}(10) \rightarrow \mathrm{SU}(5)$. The universal gaugino mass parameter $M_{1 / 2}$ at $\Lambda_{\mathcal{O}}$ is related to the universal parameter $M_{\mathcal{U}, 1 / 2}$ at $\Lambda_{\mathcal{U}}$ by

$$
M_{1 / 2}=\frac{\alpha_{\mathcal{O}}}{\alpha_{\mathcal{U}}} M_{\mathcal{U}, 1 / 2}
$$

The coefficients $\alpha_{L, R}^{\prime}$ are given by

$$
\alpha_{L}^{\prime}=\frac{3}{2} \alpha_{R}^{\prime}=\frac{36}{5 b_{5}}\left(1-\left[1+\frac{g_{S O(10)}^{2}}{16 \pi^{2}} b_{5} \log \frac{\Lambda_{\mathcal{O}}^{2}}{\Lambda_{\mathcal{U}}^{2}}\right]^{-2}\right)
$$


with $b_{5}=-3$ in the minimal $\mathrm{SU}(5)$ model including the Higgs $\{24\}$ representation, $c f$. Fig. 1(right):

$$
\begin{aligned}
b_{5} & =b_{5}(\text { matter })+b_{5}(\text { gauge })+b_{5}(\text { Higgs }) \\
b_{5}(\text { matter }) & =6 ; \quad b_{5}(\text { gauge })=-15 ; \\
b_{5}(\text { Higgs }\{24\}) & =5 ; \quad b_{5}(\text { Higgs }\{5\}+\{\overline{5}\})=1 .
\end{aligned}
$$

The $\Delta^{\prime}$ coefficients,

$$
\begin{aligned}
\Delta_{t}^{\prime} & \approx \Delta_{\nu_{\tau}}^{\prime} \approx \frac{m_{t}^{2}\left(\Lambda_{\mathcal{O}}\right)}{8 \pi^{2} v_{u}^{2}}\left(3 m_{16}^{2}+A_{0}^{2}\right) \log \frac{\Lambda_{\mathcal{O}}^{2}}{\Lambda_{\mathcal{U}}^{2}} \\
\Delta_{b}^{\prime} & \approx \frac{m_{b}^{2}\left(\Lambda_{\mathcal{O}}\right)}{8 \pi^{2} v_{d}^{2}}\left(3 m_{16}^{2}+A_{0}^{2}\right) \log \frac{\Lambda_{\mathcal{O}}^{2}}{\Lambda_{\mathcal{U}}^{2}}
\end{aligned}
$$

are (s)quark and (s)lepton loop contributions to the transport from $\Lambda_{\mathcal{U}}$ to $\Lambda_{\mathcal{O}}$. The shift $\Delta_{\lambda}^{\prime}$, accounting for contributions involving the heavy $\{24\}$ Higgs field which couples to the $\{5\}$ and $\{\overline{5}\}$ Higgs fields, is small in general; moreover, it can be neglected since the Higgs sector, in effect, plays a minor role in the analysis.

The evolution of the gauge coupling from $\Lambda_{\mathcal{U}}$ to $\Lambda_{\mathcal{O}}$,

$$
\alpha\left(\Lambda_{\mathcal{O}}\right)=\frac{\alpha\left(\Lambda_{\mathcal{U}}\right)}{1-\frac{\alpha\left(\Lambda_{\mathcal{U}}\right)}{2 \pi} b_{5} \log \frac{\Lambda_{\mathcal{O}}^{2}}{\Lambda_{\mathcal{U}}^{2}}},
$$

is affected by the Higgs- $\{24\}$ field which breaks $\mathrm{SU}(5) \rightarrow \mathrm{SM}$.

Finally, the shift of the $A$ parameters between $\Lambda_{\mathcal{U}}$ and $\Lambda_{\mathcal{O}}$ is small:

$$
\begin{aligned}
& A_{5,3} \simeq 0.97 A_{0}-0.15 M_{1 / 2} \\
& A_{10,3} \simeq 0.91 A_{0}-0.16 M_{1 / 2} \\
& A_{1,3} \simeq 0.89 A_{0}-0.10 M_{1 / 2}
\end{aligned}
$$

for the reference point defined below.

This 2-step breaking system has been analyzed quantitatively for a parameter set closely related to the previous example:

$$
\begin{aligned}
M_{0} & =100 \mathrm{GeV} & \tan \beta & =20 \\
M_{1 / 2} & =230 \mathrm{GeV} & \operatorname{sgn}(\mu) & =+ \\
A_{0} & =-425 \mathrm{GeV} & &
\end{aligned}
$$




\begin{tabular}{|l|l|l||l|l|l|}
\hline Parameter & Value & Error & Parameter & Value & Error \\
\hline \hline$m_{\tilde{e}_{R}}$ & $192.2 \mathrm{GeV}$ & $0.07 \mathrm{GeV}$ & $\mu$ & $429.5 \mathrm{GeV}$ & $5.0 \mathrm{GeV}$ \\
$m_{\tilde{e}_{L}}$ & $217.8 \mathrm{GeV}$ & $0.5 \mathrm{GeV}$ & $\tan \beta$ & 20.0 & 5.0 \\
$m_{\tilde{\nu}_{e}}$ & $202.7 \mathrm{GeV}$ & $0.7 \mathrm{GeV}$ & $M_{1 / 2}$ & $230.0 \mathrm{GeV}$ & $0.4 \mathrm{GeV}$ \\
$m_{\tilde{\tau}_{1}}$ & $101.9 \mathrm{GeV}$ & $0.3 \mathrm{GeV}$ & $A_{0}$ & $-425.0 \mathrm{GeV}$ & $8.5 \mathrm{GeV}$ \\
$m_{\tilde{\tau}_{2}}$ & $219.4 \mathrm{GeV}$ & $1.2 \mathrm{GeV}$ & & & \\
$m_{\tilde{\nu}_{\tau}}$ & $178.0 \mathrm{GeV}$ & $1.0 \mathrm{GeV}$ & & & \\
\hline
\end{tabular}

Table 3: Expected experimental errors for the determination of sfermion masses and other underlying parameters in the two-step $S O(10) \rightarrow S U(5) \rightarrow$ SM scenario. [The errors quoted correspond to $1 \sigma$.]

and

$$
\begin{aligned}
\Lambda_{\mathcal{O}} & =5 \cdot 10^{17} \mathrm{GeV} \\
D_{\mathcal{O}} & =0.9 \cdot 10^{3} \mathrm{GeV}^{2} \\
M_{\nu_{R_{3}}} & =6.3 \cdot 10^{14} \mathrm{GeV} .
\end{aligned}
$$

The scenarid 2 is compatible with values of the low-energy and cosmological data specified earlier: $\alpha_{s}\left(M_{Z}\right)=0.119, \sin ^{2} \theta_{\text {eff }}^{\text {lept }}=0.23140, \mathrm{BR}(b \rightarrow s \gamma)=3.11 \cdot 10^{-4}, \Delta a_{\mu}=25.5 \cdot 10^{-10}$, and $\Omega h_{0}^{2}=0.094$.

The analysis is performed in the same way as before, with the measurement errors extrapolated from existing studies to this scenario as listed in Tab. 3. The error on $A_{0}$ can be estimated again to be $2 \%$ according to the guideline followed in the one-step analysis, as the evolution from $\Lambda_{\mathcal{U}}$ to $\Lambda_{\mathcal{O}}$ has little impact on the $A$ parameters. Though uncertainties due to the shift of the universality scale are expected to be small, the analysis has been repeated for an error increased to $5 \%$, nevertheless. The final results are affected only slightly.

As naturally expected, a strong correlation between the D-term $D_{\mathcal{O}}$ and the $\mathrm{SO}(10)$ unification scale $\Lambda_{\mathcal{O}}$ is observed, cf. Fig. 5. Since $D_{\mathcal{O}}$ is predicted theoretically when the $\mathrm{SO}(10) \rightarrow \mathrm{SU}(5)$ breaking mechanism is devised, $D_{\mathcal{O}}$ is fixed in the following analysis while $\Lambda_{\mathcal{O}}$ is allowed to float. Results of the reverse analysis are briefly summarized for completeness.

Inspecting the solutions of the evolution equations, and Fig. 6, a simple picture emerges for

\footnotetext{
${ }^{2}$ Note that this high-scale point is outside the parameter range investigated in Ref. [31]; we thank Y. Mambrini for a clarifying comparison.
} 


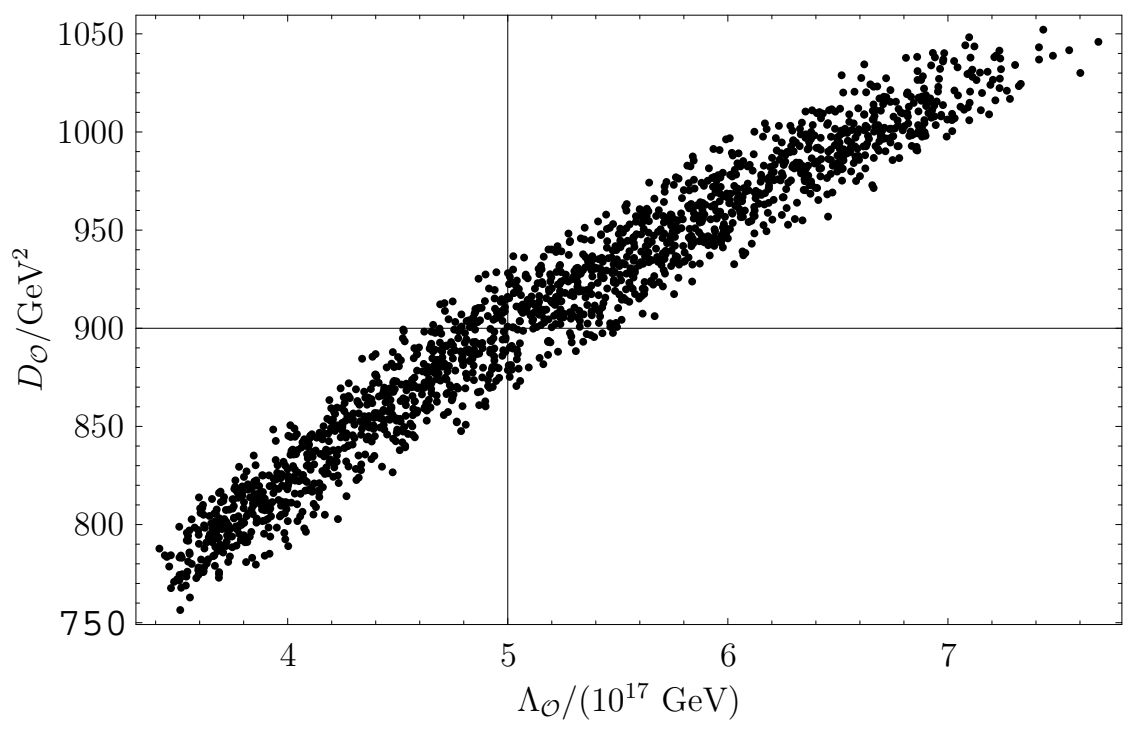

Figure 5: Correlation between the $S O(10)$ unification scale $\Lambda_{\mathcal{O}}$ and the D-term $D_{\mathcal{O}}$ in the analysis of the two-step $S O(10) \rightarrow S U(5) \rightarrow S M$ scenario. The scatter plot represents a $\chi^{2}$ analysis based on the extrapolation of the expected experimental errors from the weak scale to the unification scale.

the reconstruction of the high-scale theory:

(i) The SU(5) scale $\Lambda_{\mathcal{U}}$ can be derived from the unification point of the strong, electromagnetic and weak couplings, cf. Fig. 6(left), continuing to $\Lambda_{\mathcal{O}}$ by including the $\{24\}$ Higgs representation for $\mathrm{SU}(5)$ breaking;

(ii) The $\mathrm{SU}(5)$ mass parameters $m_{10}, m_{\overline{5}}$ are determined by the measured slepton masses of the first two generations, and the down-type scalar Higgs mass parameter analogously. Noticeable deviations from universality are observed for the mass parameters at the intermediate SU(5) GUT scale $\Lambda_{\mathcal{U}}$. [The evolution equations, Eqs. (32) and (34), depend on $S^{\prime}$, which involves the difference between the down- and up-type Higgs mass parameters, $m_{\overline{5}_{1}} m_{5_{2}}$. The corrections from the small difference of these parameters have been properly taken into account after performing the next step in the analysis based on the universality of the soft supersymmetry breaking parameters at $\Lambda_{\mathcal{O}}$ ];

(iii) The triple meeting point of the $\mathrm{SU}(5)$ parameters of the first two generations and the down-type Higgs in the evolution from the $\mathrm{SU}(5)$ to the $\mathrm{SO}(10)$ grand unification scale determines the $\mathrm{SO}(10)$ mass parameter $M_{0}$ and the GUT scale $\Lambda_{\mathcal{O}}$, cf. Fig. 6(right); 

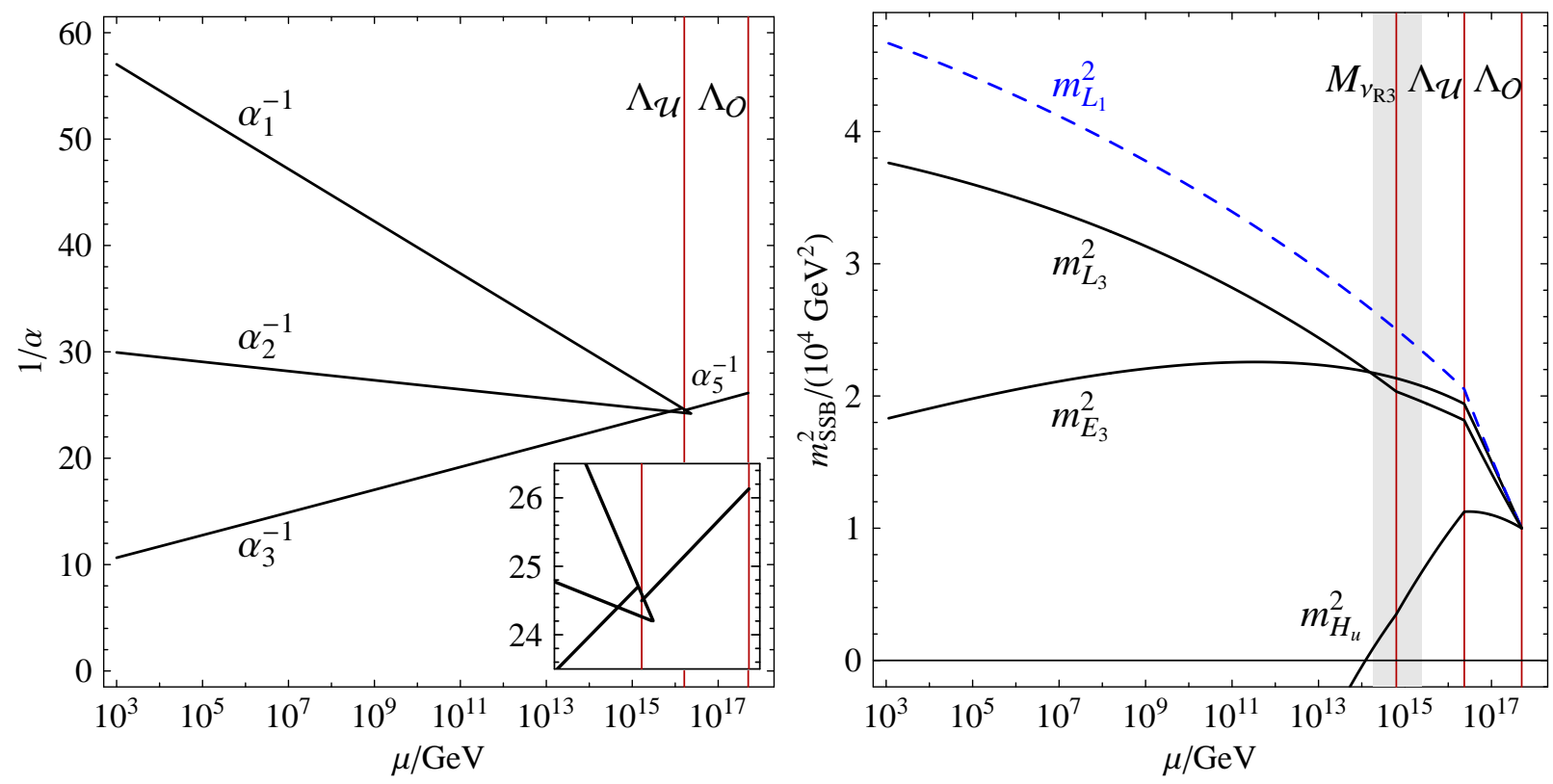

Figure 6: Left: Evolution of the gauge couplings in 2-step $\mathrm{SO}(10) \rightarrow \mathrm{SU}(5) \rightarrow$ SM symmetry breaking for the SU(5) Higgs representation \{24\}; Right: Evolution of scalar matter and down-type Higgs mass parameters, $D_{\mathcal{O}}=0$ chosen for illustration.

(iv) By matching the evolution of the slepton mass parameters in the third generation and the up-type Higgs with $M_{0}$, the value of the heavy R-neutrino mass $M_{\nu_{R 3}}$ in the seesaw mechanism can be estimated. The mass $8.6 \cdot 10^{13} \mathrm{GeV}<M_{\nu_{R 3}}<5.0 \cdot 10^{15} \mathrm{GeV}$ can only be estimated very roughly in this specific two-step scenario. This is not primarily due to the increased complexity of the 2-step structure, but rather due to the smaller value of $A_{0}$ compared to the 1-step scenario; this leads to a reduced slope in the RG evolution of $\Delta_{\nu_{\tau}}$ (cf. Fig. (4) so that the uncertainty rises in $M_{\nu_{R 3}}$. [Heavier slepton masses, however, would also reverse this tendency and reduce the error.] The uncertainty is only slightly larger, $8.0 \cdot 10^{13} \mathrm{GeV}<M_{\nu_{R 3}}<6.0 \cdot 10^{15} \mathrm{GeV}$, if the error on the $A_{0}$ parameter is increased to $5 \%$ as indicated before. If the integrated luminosities are raised to $1 \mathrm{ab}^{-1}$ both at (S)LHC and ILC, the estimate of the R-sneutrino mass narrows down to $1.8 \cdot 10^{14} \mathrm{GeV}<M_{\nu_{R 3}}<2.4 \cdot 10^{15}$ $\mathrm{GeV}$, i.e. almost by an order of magnitude. These estimates point clearly to a value in the area just below the $\mathrm{SU}(5)$ unification scale.

In this way the complete set of couplings and mass parameters could be analyzed. The results, with errors extrapolated from Tab. 3, are shown in Tab. 4. For illustration, Tab. 5 demonstrates how well the parameters can be determined when the $\mathrm{SO}(10)$ unification scale $\Lambda_{\mathcal{O}}$ is identified with the string scale while the $\mathrm{SO}(10)$ D-term is kept as a free variable. 


\begin{tabular}{|l|c|c|c|}
\hline Parameter & Mass/Scale & Error for $500 \mathrm{fb}^{-1}$ & Error for $1000 \mathrm{fb}^{-1}$ \\
\hline \hline $\mathrm{SU}(5)$ unification scale $\Lambda_{\mathcal{U}}$ & $2.35 \cdot 10^{16} \mathrm{GeV}$ & $0.02 \cdot 10^{16} \mathrm{GeV}$ & $0.02 \cdot 10^{16} \mathrm{GeV}$ \\
matter scalar mass $m_{10,1}$ & $163.3 \mathrm{GeV}$ & $0.14 \mathrm{GeV}$ & $0.10 \mathrm{GeV}$ \\
matter scalar mass $m_{\overline{5}, 1}$ & $133.5 \mathrm{GeV}$ & $0.6 \mathrm{GeV}$ & $0.45 \mathrm{GeV}$ \\
matter scalar mass $m_{10,3}$ & $142.2 \mathrm{GeV}$ & $1.2 \mathrm{GeV}$ & $0.85 \mathrm{GeV}$ \\
matter scalar mass $m_{5,3}$ & $124.2 \mathrm{GeV}$ & $0.45 \mathrm{GeV}$ & $0.4 \mathrm{GeV}$ \\
Higgs scalar mass $m_{\overline{5}_{1}}$ & $127.8 \mathrm{GeV}$ & $0.5 \mathrm{GeV}$ & $0.35 \mathrm{GeV}$ \\
Higgs scalar mass $m_{5_{2}}$ & $113.7 \mathrm{GeV}$ & $0.75 \mathrm{GeV}$ & $0.65 \mathrm{GeV}$ \\
\hline SO(10) unification scale $\Lambda_{\mathcal{O}}$ & $5.0 \cdot 10^{17} \mathrm{GeV}$ & $0.55 \cdot 10^{17} \mathrm{GeV}$ & $0.35 \cdot 10^{17} \mathrm{GeV}$ \\
D-Term $\sqrt{D_{\mathcal{O}}}$ & $30.0 \mathrm{GeV}$ & $f i x e d$ & $f x e d$ \\
matter scalar mass $M_{0}$ & $100 \mathrm{GeV}$ & $2.2 \mathrm{GeV}$ & $1.4 \mathrm{GeV}$ \\
\hline heaviest R-neutrino mass $M_{\nu_{R 3}}$ & $6.30 \cdot 10^{14} \mathrm{GeV}$ & {$[0.86,50] \cdot 10^{14} \mathrm{GeV}$} & {$[1.8,24] \cdot 10^{14} \mathrm{GeV}$} \\
lightest neutrino mass $m_{\nu_{1}}$ & $3.5 \cdot 10^{-3} \mathrm{eV}$ & {$[0.26,82] \cdot 10^{-3} \mathrm{eV}$} & {$[0.58,31] \cdot 10^{-3} \mathrm{eV}$} \\
\hline
\end{tabular}

Table 4: High-scale $S O(10)$ and $S U(5)$ parameters in 2-step $S O(10) \rightarrow S U(5) \rightarrow S M$ breaking, and masses of the heavy 3-generation $R$-neutrino and of the lightest neutrino, as reconstructed from measurements at LHC and ILC assuming a fixed value for the D-term [The errors quoted correspond to $1 \sigma]$.

\begin{tabular}{|l|c|c|c|}
\hline Parameter & Mass/Scale & Error for $500 \mathrm{fb}^{-1}$ & Error for $1000 \mathrm{fb}^{-1}$ \\
\hline \hline $\mathrm{SU}(5)$ unification scale $\Lambda_{\mathcal{U}}$ & $2.35 \cdot 10^{16} \mathrm{GeV}$ & $0.02 \cdot 10^{16} \mathrm{GeV}$ & $0.02 \cdot 10^{16} \mathrm{GeV}$ \\
matter scalar mass $m_{10,1}$ & $163.3 \mathrm{GeV}$ & $0.14 \mathrm{GeV}$ & $0.10 \mathrm{GeV}$ \\
matter scalar mass $m_{\overline{5}, 1}$ & $133.5 \mathrm{GeV}$ & $0.55 \mathrm{GeV}$ & $0.4 \mathrm{GeV}$ \\
matter scalar mass $m_{10,3}$ & $142.2 \mathrm{GeV}$ & $0.85 \mathrm{GeV}$ & $0.75 \mathrm{GeV}$ \\
matter scalar mass $m_{\overline{5}, 3}$ & $124.2 \mathrm{GeV}$ & $0.65 \mathrm{GeV}$ & $0.5 \mathrm{GeV}$ \\
Higgs scalar mass $m_{\overline{5}_{1}}$ & $127.8 \mathrm{GeV}$ & $0.22 \mathrm{GeV}$ & $0.15 \mathrm{GeV}$ \\
Higgs scalar mass $m_{5_{2}}$ & $113.7 \mathrm{GeV}$ & $0.85 \mathrm{GeV}$ & $0.7 \mathrm{GeV}$ \\
\hline SO(10) unification scale $\Lambda_{\mathcal{O}}$ & $5.0 \cdot 10^{17} \mathrm{GeV}$ & $f i x e d$ & $f i x e d$ \\
D-Term $\sqrt{D_{\mathcal{O}}}$ & $30.0 \mathrm{GeV}$ & $0.65 \mathrm{GeV}$ & $0.5 \mathrm{GeV}$ \\
matter scalar mass $M_{0}$ & $100 \mathrm{GeV}$ & $0.5 \mathrm{GeV}$ & $0.4 \mathrm{GeV}$ \\
\hline heaviest R-neutrino mass $M_{\nu_{R 3}}$ & $6.30 \cdot 10^{14} \mathrm{GeV}$ & {$[0.76,51] \cdot 10^{14} \mathrm{GeV}$} & {$[1.4,27] \cdot 10^{14} \mathrm{GeV}$} \\
lightest neutrino mass $m_{\nu_{1}}$ & $3.5 \cdot 10^{-3} \mathrm{eV}$ & {$[0.25,90] \cdot 10^{-3} \mathrm{eV}$} & {$[0.51,44] \cdot 10^{-3} \mathrm{eV}$} \\
\hline
\end{tabular}

Table 5: Same as Tab. 4, but for fixed SO(10) unification scale. 
The differences between the two sets of results are small.

The complex structure of the two-step breaking scenario, with strong correlations between some parameters, makes it impossible to derive all aspects of a general $\mathrm{SO}(10)$ scenario from measurements without making any assumption on the $\mathrm{SO}(10)$ breaking mechanism. Nevertheless, introducing assumptions about the $\mathrm{SO}(10)$ breaking as exemplified in this section, the Terascale data allow us to determine the two scales $\Lambda_{\mathcal{U}}, \Lambda_{\mathcal{O}}$, the universal scalar mass $M_{0}$, and the right-handed neutrino mass $M_{\nu_{R 3}}$ of the third generation independently. In particular, the two-step breaking scenario can be clearly distinguished from the one-step scenario analyzed in the previous section.

\section{Conclusions}

If the roots of physics are located near the Planck scale, experimental methods must be devised to explore the high-scale physics scenario including the grand unification of the Standard Model (SM) interactions up to, finally, gravity.

In this report we have studied two examples in which high-scale parameters in supersymmetric SO(10) models have been connected with experimental observations that could be expected in future high-precision Terascale experiments at LHC and $e^{+} e^{-}$linear colliders. The renormalization group provides the tool for bridging the gap between the Terascale experiments and the underlying high-scale grand unification theory. Even though it depends

on the detailed values of the parameters with which resolution the high-scale picture can be reconstructed, an accurate picture could be established in the example for one-step breaking $\mathrm{SO}(10) \rightarrow \mathrm{SM}$, including the heavy mass of the right-handed neutrino $\nu_{R 3}$ expected in the seesaw mechanism. As naturally anticipated, the analysis of two-step breaking $\mathrm{SO}(10) \rightarrow$ $\mathrm{SU}(5) \rightarrow$ SM turns out to be significantly more difficult, demanding a larger set of additional assumptions before the parametric analysis can be performed.

The two examples have demonstrated nevertheless that renormalization-group extrapolations based on high-precision data from Terascale experiments can provide essential elements for the reconstruction of the physics scenario near the grand unification if the theoretical frame is specified. 


\section{Acknowledgements}

We thank P. Bechtle, S. Wiesenfeldt and D. Zerwas for discussions, and A. Djouadi for comments on the manuscript. P. M. Zerwas thanks for the warm hospitality extended to him at RWTH Aachen and Université Paris-Sud, Orsay. This work is partially supported by the German Ministry of Education and Research (BMBF) under contract 05HT6WWA. and by the U.S. Department of Energy, Division of High Energy Physics, under Contract DE-AC02-06CH11357.

\section{References}

[1] Super-Kamiokande collaboration, Y. Fukuda et al., Phys. Rev. Lett. 81, 1562 (1998), [hep-ex/9807003]; SNO collaboration, Q. R. Ahmad et al., Phys. Rev. Lett. 89, 011301 (2002), nucl-ex/0204008; KamLAND collaboration, K. Eguchi et al., Phys. Rev. Lett. 90, 021802 (2003), hep-ex/0212021.

[2] H. Georgi, AIP Conf. Proc. 23 (1975) 575; H. Fritzsch and P. Minkowski, Nucl. Phys. B 103 (1976) 61;

[3] P. Minkowski, Phys. Lett. B 67 (1977) 421; T. Yanagida, Proceedings, Workshop on the Baryon Number of the Universe and Unified Theories (Tsukuba 1979); M. Gell-Mann, P. Ramond and R. Slansky, PRINT-80-0576-CERN; R. N. Mohapatra and G. Senjanovic, Phys. Rev. Lett. 44, 912 (1980); J. Schechter and J. W. F. Valle, Phys. Rev. D22, 2227 (1980).

[4] A. Freitas, W. Porod and P. M. Zerwas, Phys. Rev. D 72 (2005) 115002 arXiv:hep-ph/0509056.

[5] H. Baer, M. A. Diaz, P. Quintana and X. Tata, JHEP 0004 (2000) 016 arXiv:hep-ph/0002245.

[6] M. Drees, Phys. Lett. B 181 (1986) 279.

[7] C. Kolda and S.P. Martin, Phys. Rev. D53 (1996) 3871.

[8] I. Jack, D. R. T. Jones and A. F. Kord, Phys. Lett. B 579 (2004) 180 arXiv:hep-ph/0308231. 
[9] J. A. Aguilar-Saavedra et al., Eur. Phys. J. C 46 (2006) 43 arXiv:hep-ph/0511344.

[10] D. M. Pierce, J. A. Bagger, K. T. Matchev and R. J. Zhang, Nucl. Phys. B 491 (1997) 3 arXiv:hep-ph/9606211.

[11] S. Heinemeyer, W. Hollik and G. Weiglein, Eur. Phys. J. C 9 (1999) 343 arXiv:hep-ph/9812472]; G. Degrassi, P. Slavich and F. Zwirner, Nucl. Phys. B 611 (2001) 403; A. Brignole, G. Degrassi, P. Slavich and F. Zwirner, Nucl. Phys. B 631 (2002) 195; S. P. Martin, Phys. Rev. D 71 (2005) 116004 arXiv:hep-ph/0502168; Phys. Rev. D 72 (2005) 096008 arXiv:hep-ph/0509115|.

[12] G. A. Blair, W. Porod and P. M. Zerwas, Phys. Rev. D 63 (2001) 017703 arXiv:hep-ph/0007107];

[13] G. A. Blair, W. Porod and P. M. Zerwas, Eur. Phys. J. C 27 (2003) 263 arXiv:hep-ph/0210058].

[14] H. Baer, C. Balazs, J. K. Mizukoshi and X. Tata, Phys. Rev. D 63 (2001) 055011 arXiv:hep-ph/0010068].

[15] K. R. Dienes, Phys. Rept. 287 (1997) 447 [arXiv:hep-th/9602045].

[16] H. C. Cheng and L. J. Hall, Phys. Rev. D 51 (1995) 5289 [arXiv:hep-ph/9411276].

[17] E. K. Akhmedov, M. Frigerio and A. Y. Smirnov, JHEP 0309, 021 (2003) arXiv:hep-ph/0305322.

[18] S. Davidson and A. Ibarra, Phys. Lett. B 535 (2002) 25 arXiv:hep-ph/0202239; W. Buchmüller, P. Di Bari and M. Plümacher, Phys. Lett. B 547 (2002) 128 arXiv:hep-ph/0209301.

[19] J. Hisano and D. Nomura, Phys. Rev. D 59 (1999) 116005 arXiv:hep-ph/9810479].

[20] B. C. Allanach et al., in Proceedings, APS/DPF/DPB Summer Study on the Future of Particle Physics (Snowmass 2001), and Eur. Phys. J. C 25 (2002) 113 arXiv:hep-ph/0202233.

[21] A. Freitas, A. von Manteuffel and P. M. Zerwas, Eur. Phys. J. C 34 (2004) 487; A. Freitas, H. U. Martyn, U. Nauenberg and P. M. Zerwas, in Proc. of the International Conference on Linear Colliders (LCWS 04), Paris, France, 19-24 Apr 2004 hep-ph/0409129. 
[22] K. Desch, J. Kalinowski, G. Moortgat-Pick, M. M. Nojiri and G. Polesello, JHEP 0402, 035 (2004).

[23] S. Y. Choi, A. Djouadi, M. Guchait, J. Kalinowski, H. S. Song and P. M. Zerwas, Eur. Phys. J. C 14 (2000) 535 arXiv:hep-ph/0002033|; S. Y. Choi, A. Djouadi, H. S. Song and P. M. Zerwas, Eur. Phys. J. C 8, 669 (1999) [arXiv:hep-ph/9812236]; S. Y. Choi, J. Kalinowski, G. A. Moortgat-Pick and P. M. Zerwas, Eur. Phys. J. C 22 (2001) 563 [Addendum-ibid. C 23 (2002) 769] arXiv:hep-ph/0108117].

[24] G. Weiglein et al. [LHC/LC Study Group], Phys. Rept. 426 (2006) 47.

[25] P. Bechtle, K. Desch and P. Wienemann, Comput. Phys. Commun. 174 (2006) 47 arXiv:hep-ph/0412012]; P. Bechtle, K. Desch, W. Porod and P. Wienemann, Eur. Phys. J. C 46 (2006) 533 arXiv:hep-ph/0511006]; R. Lafaye, T. Plehn and D. Zerwas, arXiv:hep-ph/0512028 and arXiv:hep-ph/0404282; R. Lafaye, T. Plehn, M. Rauch and D. Zerwas, arXiv:0709.3985 [hep-ph].

[26] K. Desch, T. Klimkovich, T. Kuhl and A. Raspereza, arXiv:hep-ph/0406229.

[27] A. Bartl, H. Eberl, S. Kraml, W. Majerotto, W. Porod and A. Sopczak, Z. Phys. C 76 (1997) 549 [arXiv:hep-ph/9701336]; E. Boos, H. U. Martyn, G. A. MoortgatPick, M. Sachwitz, A. Sherstnev and P. M. Zerwas, Eur. Phys. J. C 30 (2003) 395 [arXiv:hep-ph/0303110].

[28] S. Y. Choi, H. U. Martyn and P. M. Zerwas, Eur. Phys. J. C 44 (2005) 175 arXiv:hep-ph/0508021.

[29] S. Heinemeyer, W. Hollik and G. Weiglein, Phys. Rept. 425 (2006) 265 hep-ph/0412214]; B.C. Allanach, A. Djouadi, J.L. Kneur, W. Porod and P. Slavich, JHEP 0409 (2004) 044 hep-ph/0406166]; A. Djouadi, arXiv:hep-ph/0503173; S. Heinemeyer, S. Kraml, W. Porod and G. Weiglein, JHEP 0309 (2003) 075 arXiv:hep-ph/0306181.

[30] W. Porod, Comput. Phys. Commun. 153, 275 (2003) arXiv:hep-ph/0301101].

[31] L. Calibbi, Y. Mambrini and S. K. Vempati, arXiv:0704.3518 [hep-ph]. 